\title{
Circulating Plasma MicroRNAs As Diagnostic Markers for NSCLC
}

\author{
Jinpao Hou ${ }^{1}$, Fei Meng ${ }^{1}$, Lawrence W. C. Chan ${ }^{1 *}$, William C. S. Cho ${ }^{2}$ and \\ S. C. Cesar Wong ${ }^{1}$ \\ ${ }^{1}$ Department of Health Technology and Informatics, Hong Kong Polytechnic University, Hong Kong, Hong Kong, \\ ${ }^{2}$ Department of Clinical Oncology, The Queen Elizabeth Hospital, Kowloon, Hong Kong, Hong Kong
}

\section{OPEN ACCESS}

Edited by:

Ghanshyam Upadhyay,

City College of New York, USA

Reviewed by:

Emanuele Buratti,

International Centre for Genetic

Engineering, Italy

Rajneesh Pathania

National Institute of Environmental

Health Sciences $(N I H)$, USA

Nagaraja Nagre,

Eastern Virginia Medical School, USA Shinjini Singh,

University of Texas MD Anderson

Cancer Center, USA

*Correspondence:

Lawrence W. C. Chan

wing.chi.chan@polyu.edu.hk

Specialty section:

This article was submitted to

RNA,

a section of the journal

Frontiers in Genetics

Received: 05 July 2016 Accepted: 18 October 2016 Published: 03 November 2016

Citation:

Hou J, Meng F, Chan LWC, Cho WCS and Wong SCC (2016) Circulating Plasma MicroRNAs As Diagnostic

Markers for NSCLC

Front. Genet. 7:193.

doi: 10.3389/fgene.2016.00193
Lung cancer is the most common cause of cancer deaths all over the world, in which non-small cell lung cancer (NSCLC) accounts for $\sim 85 \%$ of cases. It is well known that microRNAs (miRNAs) play a critical role in various cellular processes, mediating post-transcriptional silencing either by mRNA degradation through binding the $3^{\prime}$ UTR of target mRNA or by translational inhibition of the protein. In the past decade, miRNAs have also been increasingly identified in biological fluids such as human serum or plasma known as circulating or cell-free miRNAs, and may function as non-invasive diagnostic markers for various cancer types including NSCLC. Circulating tumor cells (CTCS) are those cells that are shed from solid tumors and then migrate into the circulation. However, reports concerning the roles of CTCs are quite rare, which may be attributed to the difficulties in the enrichment and detection of CTCs in the circulation. Although, there have been reassuring advances in identifying circulating miRNA-panels, which are assumed to be of diagnostic value in NSCLC early stage, some issues remain concerning the reliability of using miRNA panels as a diagnostic tool for NSCLC. In the current review, we are aiming at providing insights into the miRNAs biology, the mechanisms of miRNAs release into the bloodstream, cell-free miRNAs as the diagnostic markers for NSCLC and the current limitations of CTCs as diagnostic markers in NSCLC.

Keywords: NSCLC, circulating miRNAs, miRNA panel, diagnostic value, CTCs detection

\section{INTRODUCTION}

Lung cancer is the most common cause of cancer deaths in men and the second leading cause of cancer deaths in women (Jemal et al., 2010; Ozretić et al., 2012). WHO has shown that lung cancer strikes over 1,605,000 people each year and explains $18.2 \%$ of all cancer-related deaths (http:// globocan.iarc.fr). There are two types of lung cancer: small cell lung cancer (SCLC) that is a more aggressive type and accounts for $15 \%$ of cases, and non-small cell lung cancer (NSCLC), which comprises small squamous cell carcinoma (SCC), adenocarcinoma (AD) and large cell carcinoma (LCC). Remarkably, NSCLC alone accounts for $85 \%$ of cases (Travis et al., 2011; Wood et al., 2012). With respect to NSCLC, the main reason for the current high mortality is its late diagnosis and poor prognosis. According to the data from National Cancer Institute, 55\% of cases are diagnosed at an advanced stage and over half of the lung cancer patients die within one year of diagnosis (SEER Cancer Statistics Review). In addition, the 5-year survival rates vary from $67 \%$ in Stage IA to $39 \%$ in Stage IIB, and for patients who were at inoperable advanced stage, the 5-year survival rates even hardly reach 3.3\% (Scagliotti et al., 2003; Jemal et al., 2004). Traditional diagnosis of NSCLC tends to be based on either computed tomography (CT) scans or chest X-ray following 
histological examination of the tissue. Unfortunately, it has been estimated that less than $30 \%$ of cases can be detected at an early stage when the curative surgery is possible. The possible reason may be a lack of reliable biological markers indicative of lung cancer at earlier stage when tumors are surgically resectable. Thus far more cases diagnosed are already at an advanced stage when conventional surgical resection of tumor is impractical due to the emergence of tumor metastasis. Furthermore, for various reasons, the tumor tissues tend to be less attainable in clinical practice. Consequently, it is imperative to find a minimally invasive marker for NSCLC early diagnosis, thus improving the prognosis of this disease.

MiRNAs are endogenous small non-coding RNA molecules, 19-22 nucleotides in length, and function as regulatory molecules mediating post-transcriptional silencing either by the promotion of mRNA degradation or by the inhibition of protein translation. The first miRNA was identified in C. elegans (Lee et al., 1993). It is well known that miRNAs play a critical role in virtually all signal pathways in various tumor types. With respect to NSCLC, a vast number of reports have shown that the difference between miRNA expression profiles in NSCLC tissues or cell lines and those in healthy controls might be of diagnostic value for NSCLC. For instance, Raponi and colleagues have identified 15 differentially expressed miRNAs between SCCs and healthy lung tissues, among which let-7e and miR-125a were downregulated while the remaining 13 miRNAs were upregulated (Raponi et al., 2009). Similarly, another study by Petriella and coworkers have examined the diagnostic power of miRNAs measurements in fine-needle aspiration NSCLC biopsies and revealed that three miRNAs (miR-7, miR-21, miR-155) exhibited a higher level in tumoral FNA when compared with normal FNA specimens, while let-7a exhibited a lower level (Petriella et al., 2013). Overall, one ambitious aim of these researchers is to develop a reliable miRNA-based method as a convenient tool for the early diagnosis of lung cancer. However, the inevitable invasiveness of using resected tumor samples and the unavailability of tumor tissues limit its routine clinical application. Indeed, not all lung cancer patients have operable diseases and many of them do not have available tumor tissues for genetic analysis (Gao et al., 2011). By contrast, circulating miRNAs in biological fluids such as plasma are emerging as a non-invasive biomarker for NSCLC diagnosis. Most importantly, these non-invasive biomarkers are valuable for predicting drug response by monitoring genetic profiles during treatment, which holds great potential for personalized therapy.

In 2008, 2 research groups reported that human plasma or serum contain a huge amount of miRNAs existing in fairly stable forms, and that these miRNAs profiles hold great promise as novel non-invasive markers for the early diagnosis of cancers (Chen et al., 2008; Mitchell et al., 2008). Since then, reports focusing on the roles of circulating miRNAs in NSCLC have gradually increased, of which the vast majority are involved in determining the cell-free miRNAs panels that can discriminate

Abbreviations: MVB, Microvesicular bodies; AGO, Argonaute proteins; MiRISC, miRNA-induced silencing complex; $3^{\prime}$ UTR, 3'untranslated region; Ldbr, lariat debranching enzyme; KSRP, KH-type splicing regulatory protein; hnRNP A1, heterogeneous nuclear ribonucleoprotein A1.
NSCLCs from healthy controls. Apparently, the analysis of circulating miRNA expression profiles has obvious advantages over that of tissue or cell line-derived miRNAs.

Circulating tumor cells (CTCs), are a group of cells that are shed from solid tumors and then migrate into the circulation. They are widely assumed to be responsible for tumor metastasis (O'Flaherty et al., 2012; Parkinson et al., 2012). Although, CTCs are rare in peripheral circulation, it seems to be attractive to use CTCs as a diagnostic marker of NSCLC, especially when obtaining adequate tissue from patients for diagnosis is difficult. The rarity of CTCs necessitates a more sensitive and more specific detection technique. However, only a few studies reported thus far focus on CTC detection and enumeration, most of which just confirmed the intimate relation between high CTCs numbers and poor prognosis (Hou et al., 2009; Jorge et al., 2012). With regard to CTC-associated miRNAs, only a few studies have been conducted currently to define the subpopulation of CTCs associating it with the prognosis of NSCLC. In the current review, we reviewed miRNAs biology, the mechanisms of miRNAs release into the bloodstream, cell-free miRNAs as diagnostic markers for NSCLC, and the current limitations of CTCs as diagnostic markers in NSCLC.

\section{MIRNA BIOSYNTHESIS AND MODE OF ACTION}

There are mainly two different pathways involved in miRNA biosynthesis: canonical pathway and non-canonical pathway. The canonical pathway involves a step-wise process starting from the nucleus and finishing in the cytoplasm, in which various enzymes and accessory proteins participate. Firstly, long primary miRNA (pri-miRNA) transcript is transcribed by RNA polymerase II (Bartel and Chen, 2004). Then, the resulting primiRNA are processed by nuclear RNase III enzyme Drosha and a cofactor Digeorge syndrome critical region 8 (DGCR8), thus producing pre-miRNA of $\sim 70 \mathrm{nt}$ in length (Lund et al., 2004). Subsequently, the nuclear export of pre-miRNA by Exportin5 into the cytoplasm allows nuclease Dicer cleavage, yielding a paired $\sim 22 \mathrm{nt} \mathrm{miRNA/miRNA*}$ duplex. Finally, one strand of the duplex, namely mature miRNA, is loaded into a protein complex containing Argonaute (AGO) to assemble the RNA-induced silencing complex (RISC) responsible for gene silencing, with the remaining strand known as miRNA* undergoing degradation. Afterwards, RISC guided by the mature miRNA binds to the $3^{\prime}$-UTR of target mRNAs resulting in mRNAs degradation or protein synthesis inhibition (Krol et al., 2010). RISC recognizes its target mRNAs by Watson-Crick base pairing between miRNAs and the "seed sequences" of target mRNA sites.

However, some miRNAs are derived from short hairpin introns called "mirtrons," the biosynthesis of which involves in a non-canonical pathway. This is a splicing-dependent and Drosha-independent mechanism of miRNA biosynthesis as the primary transcript is spliced and then debranched by lariat debranching enzyme (Ldbr) to form pre-miRNA as a Dicer substrate, which bypasses Drosha/DGCR8 processing (Westholm and Lai, 2011). It is notable that recent studies also have 
found $5^{\prime}$ and $3^{\prime}$ tailed mirtrons that are very similar to conventional mirtrons. The difference between them is that following debranching, tailed mirtrons contain a single-stranded tail on either $5^{\prime}$ or $3^{\prime}$ of the pre-miRNA-like hairpins, which needs to be trimmed by an exonuclease known as RNA exosome to produce pre-miRNAs for Dicer cleavage (Okamura et al., 2008; Westholm and Lai, 2011). In effect, pre-miRNAs derived from both conventional miRNAs and mirtrons or tailed mirtrons are further exported by Exportin-5 into the cytoplasm, where they are subject to Dicer cleavage to generate miRNA duplex (Figure 1).

Several RNA-binding proteins are involved in a select group of miRNA biosynthesis, acting either as "co-activators" or "co-repressors" for the processing of miRNA precursors. Among them are the three most important proteins: Cell lineage abnormal 28 (Lin-28), KH-type splicing regulatory protein (KSRP) and heterogeneous nuclear ribonucleoprotein A1 (hnRNP A1). Numerous studies have demonstrated that human paralogs Lin-28a and Lin-28b can prevent the maturation of let-7 by interacting with pri- or pre-let-7 via the terminal loop (TL) elements to inhibit their processing by Drosha and Dicer (Choudhury and Michlewski, 2012; Huang, 2012; Mayr and Heinemann, 2013). Intriguingly, Lin-28 protein levels are found to be much higher in undifferentiated cells and the early stages of embryonic development, while mature let-7 miRNAs are absent despite the existence of pre-let-7 (Choudhury and Michlewski, 2012). By contrast, KSRP, also known as FBP2, are shown to act as an auxiliary protein to promote the maturation of select miRNAs including let-7 by binding to TL elements and interacting with Drosha and Dicer, which is similar to the way Lin-28 bind to miRNA precursors (Gherzi et al., 2010). In addition, hnRNP A1 is one of the most abundant members in the protein family of hnRNPs. Unlike lin-28 or KSRP, hnRNP A1 either promotes or inhibits the maturation of miRNAs via different mechanisms according to the miRNA precursor it interacts with. For example, Michlewski G and co-workers showed that hnRNP A1 is required for the processing of pri-miR-18a by binding to its TL element to induce stem-loop structure rearrangement, thus facilitating more effective Drosha cleavage (Michlewski et al., 2008; Choudhury and Michlewski, 2012). However, these authors also have revealed that hnRNP A1 binds to the TL element of pri-let-7a to inhibit Drosha cleavage, thus preventing the processing of pri-let-7a. Most interestingly, they also confirmed the same sequence in the TL element of pri-let-7a is the target of KSRP and proposed that hnRNP A1 and KSRP play antagonistic roles in the maturation of let-7a (Michlewski and Cáceres, 2010). The roles of these protein factors in the processing of miRNA precursors are shown in Figure 1.

\section{POSSIBLE MECHANISMS OF MIRNA ENTERING INTO THE CIRCULATION}

As non-invasive serological markers of tumors, circulating miRNAs are first identified concurrently by two groups in 2008 . Mitchell et al. have demonstrated that circulating miRNAs levels in human plasma remain stable even when plasma is subjected to prolonged incubation at room temperature. In addition, they showed that circulating miRNAs are resistant to multiple freezethaw cycles. Furthermore, they used a mouse model to reveal that extracellular miRNAs arising from human prostate cancer xenografts enter the circulatory system (Mitchell et al., 2008). By Solexa sequencing, Chen et al. (2008) found that compared to healthy donors, 28 miRNAs species are missing and 63 new miRNA species are detected in lung cancer patients, suggesting that human serum might contain miRNA signatures of the ongoing disease (Chen et al., 2008). These two studies established the concept that one can diagnose cancers based on specific cellfree miRNA signatures. In the meantime, a number of intriguing questions have been posed, among which are the routes of miRNA release into the circulation.

During the past decade, several research groups have found that circulating miRNAs can be packaged into some types of membrane-bound vesicles such as microvesicular bodies (MVB) or exosomes. Given the remarkable stability of circulating miRNA, for several years, the predominant view was that the vast majority of cell-free miRNAs were released from cells in vesicles (Valadi et al., 2007; Zernecke et al., 2009; Collino et al., 2010). However, others have revealed that cell-free miRNAs also exist in vesicle-free forms associating with protein complexes including high and low density lipoproteins and RNA-binding proteins (Wang et al., 2010; Arroyo et al., 2011; Vickers et al., 2011). For instance, Arroyo JD have shown that Argonaute2 (AGO2), the key effector protein of miRNA-mediated silencing, is present in human plasma and serve as a significant carrier of circulating miRNAs in plasma as shown in Figure 1. They also found that potentially $90 \%$ of circulating miRNAs are present in vesicle-free form, which suggest that circulating miRNA biomarker analysis based only on exosome purification might be ineffective for miRNA biomarkers that circulate as nonexosomal AGO2 complexes (Arroyo et al., 2011). Additionally, Vicker KC et al. have demonstrated that high-density lipoprotein particles (HDL) transport endogenous miRNAs and deliver them to recipient cells, the process of which is regulated by neutral sphingomyelinase and dependent on scavenger receptor class B type I (Vickers et al., 2011). Moreover, vesicle-associated miRNAs versus vesicle-free miRNAs may originate from different cell types and indicate cell-type specific miRNA release mechanisms. For example, miR-122, a liver-specific miRNA, circulates only in protein-associated form, suggesting that hepatocytes release miR122 via protein carrier pathway (Chang et al., 2004). By contrast, miRNAs primarily present in membrane-bound vesicles, such as let-7a, may originate from cell types that generate vesicles (Arroyo et al., 2011). However, the relative abundance of vesiclederived miRNAs versus vesicle-free miRNAs for any given miRNA species remains to be explored.

\section{CIRCULATING MIRNAS AS DIAGNOSTIC BIOMARKERS OF NSCLC}

The non-invasiveness, stability and reproducibility make circulating miRNAs an ideal diagnostic marker in oncology and hold great promise for clinical applications. Since circulating 


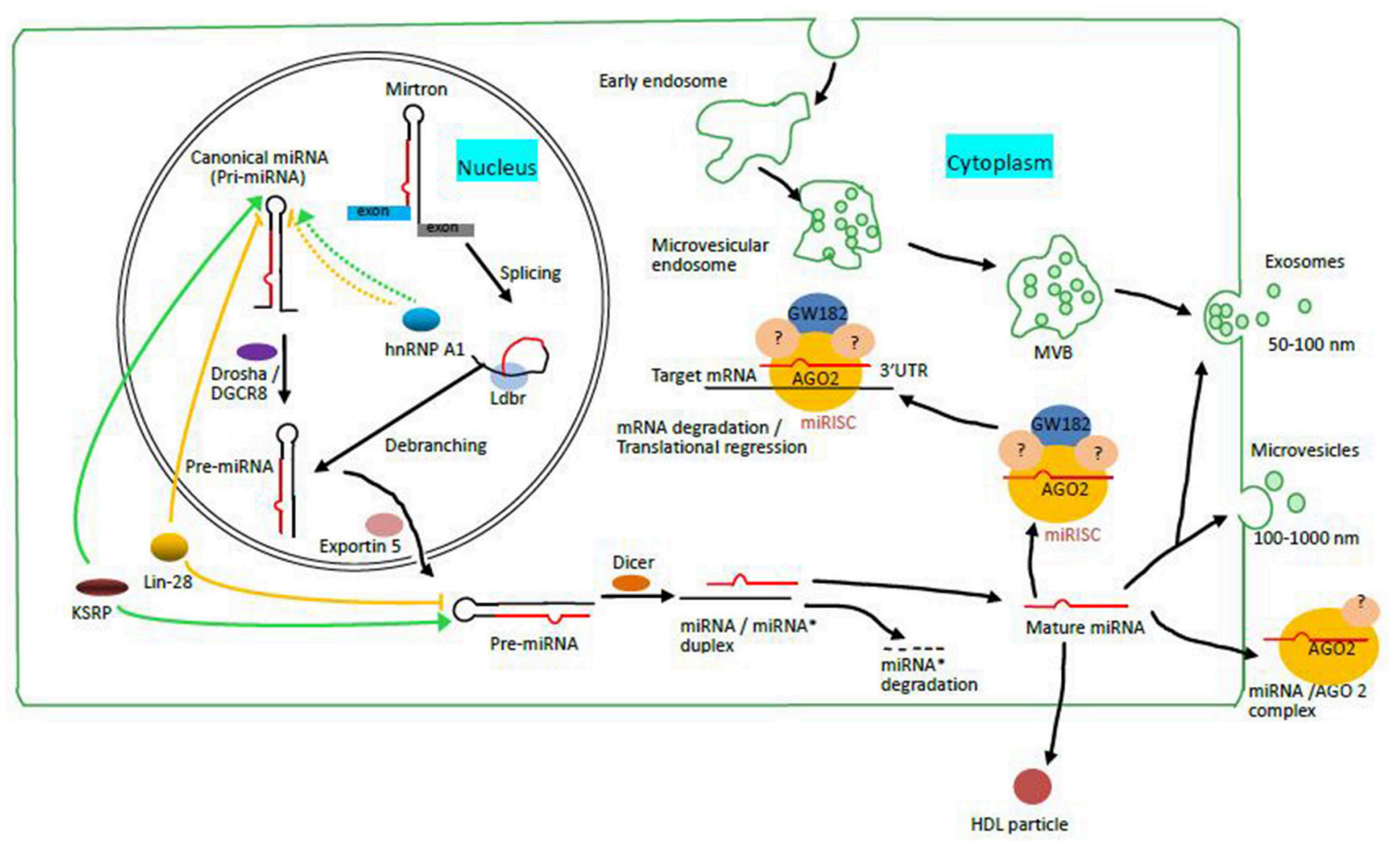

FIGURE 1 | MiRNA biosynthesis through canonical and non-canonical pathways and the mechanisms of miRNA release into the bloodstream (Modified from Zandberga et al., 2013). Mirtrons are spliced, and then debranched by lariat debranching enzyme (Ldbr) to generate pre-miRNAs, the product of Drosha/DGCR 8 cleavage of pri-miRNAs in canonical pathways. Pre-miRNAs from both pathways are exported into the cytoplasm by Exportin 5 for further processing by Dicer, thus producing double-stranded miRNA/miRNA* duplexes. Subsequently, one strand of this duplex is loaded into miRNA induced silencing complex (miRISC) containing one of four AGOs (such as AGO2 as shown), GW182 and various unknown GW182-interacting silencing effectors, leaving the other strand undergoing degradation. The mature miRNA then guides the miRISC to bind to the $3^{\prime}$ UTR of target mRNAs either to promote mRNA degradation or to inhibit protein translation. KSRP as a co-activator and Lin-28 as a co-repressor bind to the terminal loop (TL) elements of pri or pre-miRNAs to promote and inhibit the maturation of a subset of miRNA precursors respectively. HnRNP A1 can both promote (for pri-miR-18a) and inhibit (for pri-let-7a) the processing of miRNAs by binding to the TL elements of pri-miRNAs as shown in the figure, which is mediated by different mechanisms. Circulating miRNAs exist either as a vesicle-associated form or as a protein-associated form in the circulation. The former (vesicles) include exosomes and microvesicles. The latter (proteins) include high and low density lipoproteins, RNA binding proteins such as Argonaute 2.

miRNAs were first identified in human plasma in 2008, a great many of studies have been aiming at identifying a reliable diagnostic tool by investigating the differential expression of circulating miRNAs between NSCLC patients and healthy controls or patients harboring benign tumors. For instance, Lihong Fan et al. have demonstrated that a predictive model combining serum miR-15b-5p, miR-16-5p, miR-20a-5p can be used to discriminate the early stage of NSCLC cases from healthy subjects (Fan et al., 2015). Reassuringly, the fact that this study applied two different methods (RT-qPCR and Nanoquantum dots microarray) to identify the same differential expression of serum miRNAs, which may have improved the sensitivity and reproducibility of the results. Additionally, Li $\mathrm{Y}$ et al. have isothermally sensitively detected lung cancerassociated serum miR-486-5p through hairpin probe-based rolling circle amplification (HP-RCA) with superhigh sensitivity (Li et al., 2013). In practice, miR-486-5p has been shown to be downregulated in both the primary cancer and the serum of
NSCLC patients in several independent research groups (Volinia et al., 2006; Boeri et al., 2011; Shen et al., 2011), which suggests this miRNA might serve as a tumor suppressor involved in multiple signaling circuits in NSCLC and thus deserves highly attention in future study. Furthermore, a 34-miRNA panel in serum has been identified in patients at the early stage of NSCLC in a group of asymptomatic high-risk subjects with up to $80 \%$ accuracy (Bianchi et al., 2011). The signature obtained in this study allows the diagnosis of asymptomatic patients instead of symptomatic ones diagnosed by other signatures in many previous studies. Most importantly, it is specific for lung cancer compared with breast cancer. However, the researchers of this study may well test for the specificity of this signature in other more cancer types other than breast cancer before its clinical application. Moreover, using three different analytical methods, A. Markou and colleagues identified an eight miRNA-panel which can be used for discriminating cancerous tissues from non-cancerous ones in NSCLC patients. By contrast, only 
three of these miRNAs (miR-30e-5p, miR-21 and miR-10a) are differentially expressed in NSCLC plasma specimens compared to that from healthy plasma, suggesting miRNA profiles in the circulation may not necessarily reflect exactly those in tumor tissues (Markou et al., 2013). However, an earlier report have showed that miRNA footprints did not show significant difference between circulating exosome-derived miRNAs and the tumoral miRNAs profiles using 4 lung cancer tissues and their corresponding plasma samples (Rabinowits et al., 2009). This contradiction between these two studies may be attributed to two factors: one is that the internal control gene used for the normalization of miRNA expression may vary between different research groups; the other is that circulating miRNAs do not exist only in exosomes as mentioned earlier, most of them are shown to be associated with protein complexes. Most importantly, only 4 lung cancer tissues used for the study are not enough to represent a reliable result.

In the past two years, a vast number of efforts have been made to identify circulating miRNA panels for NSCLC early diagnosis. However, many of these studies remain to be validated given the inconsistency of reference controls used for the normalization and of the materials such as exosomes, serum and whole blood used for RNA isolation. For instance, Magdalena B. Wozniak and colleagues showed that a 24-miRNA panel alone could distinguish lung cancer patients from healthy controls with an AUC of 0.92 , and they also revealed that this diagnostic power can be further enhanced by adding factors such as age, sex and smoking status into this model (Wozniak et al., 2015). Similarly, 6 cell-free miRNAs (200b, 429, 205, 125b, 34b, 203) have also been validated with an even higher abundance in the serum of NSCLC patients compared to healthy subjects (Halvorsen et al., 2016). However, a common problem in these two studies is that the authors have chosen U6 as the internal control for the normalization when analyzing the miRNAs profiles, which may be inaccurate as U6 has been reported to be not a suitable endogenous control for the quantification of cell-free miRNAs (Benz et al., 2013; Xiang et al., 2014). Therefore, it is conceivable that the conclusions obtained from those studies should be interpreted with caution. In addition, Silva et.al reported that the levels miR-30e-3p, let-7f and miR-20b, were downregulated in the plasma vesicles of NSCLC cases compared with healthy controls (Silva et al., 2011). Another similar study showed that a panel of miRNAs $(30 b, 30 c, 103,122,195,203,221,222)$ in exosomes from the plasma displayed significantly differential expression between NSCLC cases and healthy donors, which might suggest the possibility that miRNAs signatures from patient exosomes could alone represent the status of ongoing disease (Giallombardo et al., 2016). However, these studies might not be complete as researchers focused only on vesicle-associated miRNAs, such as exosomes, probably missing the differential expression of vesicle-free miRNAs that may represent a larger proportion of miRNAs in the circulation and thus producing an inaccurate result. Most of the reported results identifying circulating miRNA panels as diagnostic biomarkers in the last two years are summarized in following Table 1. As shown in the table, different miRNA-panels have been identified by different research groups. Actually, some above mentioned factors such as various biological materials and different internal controls used may account for this phenomenon.

Although, there do exist some concerns about current studies of circulating miRNAs, some critical miRNAs as tumor suppressors or oncogenes are of great value to develop miRNAbased strategy for lung cancer therapy and therefore should be prioritized to further studies with respect to their diagnostic power. Among these critical miRNAs, miR-21 is a known to be an "oncomir" and is commonly overexpressed in cancers including lung, breast and colorectal cancer (Ma et al., 2014). For example, Si ML and colleagues showed that miR-21 inhibition suppresses tumor growth in xenograft mouse model and increases cell apoptosis that is associated with downregulation of bcl-2 expression in breast cancer (Si et al., 2007). Also, higher expression of miR-21 and miR-155 in both tumor tissues and serum can predict recurrence and poor survival in NSCLC (Yang et al., 2013). This may suggest that overexpression of circulating miR-21 holds great prognostic potential in NSCLC. Another aspect about miR-21 is that it influences response to chemotherapy in several tumor types and thus can act as a therapeutic target for overcoming drug resistance in cancers. For instance, a recent study has revealed that antisense inhibition of miR-21 or miR-221 sensitizes the effects of Gemcitabine, a chemotherapeutic treatment of pancreatic cancer (Park et al., 2009). By contrast, let-7 family miRNAs are known as tumor suppressors and down-regulation of let-7 expression has been demonstrated in breast, prostrate, ovarian and lung cancers (Boyerinas et al., 2010). Dejuan Kong et al. have provided evidence that let-7 loss mediates up-regulation of EZH2 and contributes to PCa aggressiveness, which can be attenuated by BDIM, a potent agent in inhibiting the growth of Pca cells (Kong et al., 2012). In addition, numerous studies have revealed that let-7 acts as a promising therapeutic target for lung cancer. For example, Trang.s group have shown that systematical delivery of synthetic let-7 mimic or miR-34a into lung tumor-bearing mice results in significant tumor regression compared to the delivery of a miRNA control (Trang et al., 2011). Overall, since current studies on the roles of miR-21 and let-7 family in various cancer types have mainly focused on tumor tissues, circulating miR-21 and let-7 in plasma warrant further validation before applying them as biomarkers for the early diagnosis of cancers including NSCLC.

\section{CURRENT LIMITATIONS ON CTCS AS DIAGNOSTIC MARKERS OF NSCLC}

CTCs are tumors cells which are released from solid tumors, and then migrate into the circulation and play a critical role in tumor metastasis. However, CTCs are extremely rare, and they can be detected at a low frequency of 1 CTCs per $10^{6}-10^{7}$ leukocytes (Young et al., 2012). Initially, CTCs were considered as non-leukocytic cells with epithelial origin since they have been detected in various epithelial cancers such as breast cancer, lung cancer and colon cancer (Allard et al., 2004). Consequently, epithelial marker-based methods for the detection of CTCs have been widely used (Hardingham et al., 1993; Liljefors et al., 2005). 
TABLE 1 | Circulating miRNAs as diagnostic markers for NSCLC.

\begin{tabular}{|c|c|c|c|c|c|}
\hline Significantly-expressed miNRAs & Scope & Sample & Technique & Normalization & References \\
\hline let-7c, miR-152 (down) & 120 NSCLCs vs. $360 \mathrm{HCs}$ & Plasma & qRT-PCR & U6 & Dou et al., 2015 \\
\hline $\begin{array}{l}\text { miR-16-5p, miR-17b-5p, miR-19-3p, } \\
\text { miR-20a-5p, miR-92-3p (down) } \\
\text { miR-15b-5p(up) }\end{array}$ & $\begin{array}{l}\text { Training set: } 94 \text { NSCLCs vs. } 58 \mathrm{HCs} \text {, } \\
\text { Validation set: } 70 \text { NSCLCs vs. } 54 \mathrm{HCs}\end{array}$ & Serum & TaqMan miRNA assays & $\begin{array}{l}\text { Absolute } \\
\text { quantification }\end{array}$ & Fan et al., 2015 \\
\hline $\begin{array}{l}\text { miR-148/152 family (Down) miR-944, } \\
\text { miR-3662 (up) miR-483-5p, miR-193a-3p, } \\
\text { miR-25, miR-214 miR-7 (up) }\end{array}$ & $\begin{array}{l}20 \text { NSCLCs with BPD VS. } 10 \text { HCs } 90 \\
\text { NSCLCs vs. } 85 \text { HCs } 221 \text { NSCLCs, } \\
161 \text { HCs, } 56 \text { with benign nodules }\end{array}$ & $\begin{array}{l}\text { Serum } \\
\text { Plasma }\end{array}$ & $\begin{array}{l}\text { RT-qPCR RT-qPCR } \\
\text { TaqMan Low Density Array, } \\
\text { RT-qPCR }\end{array}$ & $\begin{array}{l}\text { U6 U6 let- } 7 \mathrm{~d} / \mathrm{g} / \mathrm{i} \\
\text { trio }\end{array}$ & $\begin{array}{l}\text { Chen et al., 2015; } \\
\text { Li et al., 2015; } \\
\text { Powrózek et al., } \\
2015\end{array}$ \\
\hline miR-125a-5p, miR-145 miR-146a (up) & 70 NSCLCs vs. 70 HCs & Serum & RT-qPCR & miR-39 & Wang et al., 2015 \\
\hline $\begin{array}{l}\text { A panel of } 24 \text { miRNAs (Relative } \\
\text { expression) }\end{array}$ & 100 NSCLCs vs. $100 \mathrm{HCs}$ & Plasma & TaqMan MiRNA Arrays & $\begin{array}{l}\text { U6snRNA } \\
\text { ath-miR-159a }\end{array}$ & $\begin{array}{l}\text { Wozniak et al., } \\
2015\end{array}$ \\
\hline A panel of 8 miRNAs (Relative expression) & 12 NSCLCs vs. 6 HCs & Exosomes & RT-PCR & mir-1228-3p & $\begin{array}{l}\text { Giallombardo } \\
\text { et al., } 2016\end{array}$ \\
\hline
\end{tabular}

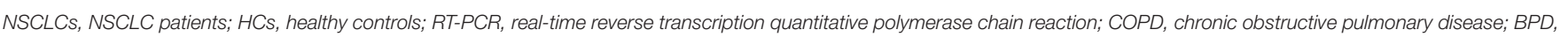
benign pulmonary diseases.

Nevertheless, recent studies have shown that the morphological characteristics of CTCs have yet to be well defined and may vary according to tumor stages (Parkinson et al., 2012). Overall, more clear phenotypes of CTCs as a "liquid biopsy" remain to be explored in future study.

As a "liquid biopsy," current studies on CTCs as a biomarker of NSCLC have been limited to its enrichment and detection as well as its relation to NSCLC prognosis. For instance, Laura Muinelo-Romay and colleagues found that patients with CTCs $>5$ are assumed to correlate with poorer prognosis based on CTCs counts using CellSearch technology (Muinelo-Romay et al., 2014). Another group have isolated CTCs by cell size filtration and shown that CTCs can be identified in two-thirds of patients at advanced stage of NSCLC at diagnosis, suggesting that CTCs counts may reflect the ongoing disease status of NSCLC (Mascalchi et al., 2016).

However, less progress has been made in the field of CTCs as an ideal biomarker for NSCLC diagnosis, and this may result from several aspects. For one thing, various techniques for CTCs enrichment and later detection have been developed which base either on physical properties such as size, density and electric charges, or on biological properties such as the expression of CTCs surface marker (Alix-Panabieres and Pantel, 2013), among which the most widely used method relies on the epithelial protein, namely, Epithelial cell adhesion molecule (Ep-CAM) for the CTCs enrichment (Allard et al., 2004; Harb et al., 2013; Haus et al., 2014). However, Hanssen A et al. reported that the expression Ep-CAM may be downregulated in CTCs experiencing epithelial-to-mesenchymal transition (EMT), which raises the concern that this Ep-CAM based CTCs enrichment method may lose EMT-associated CTCs (Hanssen et al., 2016). Additionally, another group, Yingchun Man et.al have developed four CTCs markers (CK7, CLCA2, HMMR and hTERT) to detect CTCs, which significantly improves the specificity and sensitivity of CTC detection (Man et al., 2014). Intriguingly, by miRNA in situ hybridization, Francisco G.
Ortega et al. have identified miR-21 within CTCs as a marker used for detecting CTCs displaying an EMT phenotype (Ortega et al., 2015). We can infer that miR-21 may be function as an oncomir implicated in tumor metastasis. In a nutshell, whether epithelial maker-dependent or -independent approach is more sensitive and specific in CTCs enrichment and detection remains to be explored. For another thing, CTCs counts alone may not fully represent the ongoing NSCLC status especially at a very early stage. This concept is clearly intelligible given that several results have shown CTCs heterogeneity as a diagnostic marker by identifying different CTCs subpopulations. In a research reported by $\mathrm{Wu}$ et al. (2015), three CTCs subpopulations, epithelial CTCs, biophenotypic epithelial/mesenchymal CTCs and mesenchymal CTCs, were identified using epithelial-tomesenchymal transition markers, which is useful to identify the more aggressive CTC phenotypes and thus facilitates the determination of clinical practice.

\section{DISCUSSION}

NSCLC diagnosis, especially at early stage is in great demand in clinical practice. Conventional diagnostic applications relying on tissue biopsy either through computed tomography scans or chest X-ray have remarkable limitations given their low sensitivity and invasiveness as well as tissue unavailability. Consequently, a novel, reliable and reproducible diagnostic biomarker is badly needed to obtain an earlier and more accurate diagnosis result.

Circulating miRNAs in biological fluids, especially in human plasma or serum, have emerged as a non-invasive diagnostic marker for NSCLC. In the past five years, studies in this field have identified different miRNA panels in the circulation with higher sensitivity plus higher specificity in early NSCLC diagnosis, which might be preferable compared to any single miRNA used for NSCLC diagnosis. In addition, several factors 
may contribute to the fact that different miRNA panels have been identified for NSCLC diagnosis. To begin with, different materials such as plasma/serum, whole blood, or exosomes used for cell-free miRNAs isolation make it possible that the quality and quantity of circulating miRNAs isolated by different researcher vary according to biological material types. Indeed, whole blood also contains a large amount of peripheral blood mononuclear cells (PBMCs) compared with serum or plasma. Intriguingly, a two-miRNA panel (miR-19b-3p and miR-29b$3 p$ ) arising from PBMCs have been developed by Jie Ma et al. to discriminate NSCLCs from healthy persons with $72.62 \%$ sensitivity and $82.61 \%$ specificity. This group also identified SCC, the major type of NSCLC using this two-miRNA panel, with $80.00 \%$ sensitivity and $89.86 \%$ specificity, which may imply that only focusing on serum-derived circulating miRNAs may not be enough to reflect a reliable result in terms of NSCLC diagnosis based on circulating miRNA detection (Ma et al., 2015). In addition, the inconsistency of the methods used for data analysis should also be considered to account for the existence of various miRNA-panels. As shown in Table 1, internal controls such as U6 and miR-39 have been used for the normalization of miRNA expression. Unfortunately, the high variability of non-coding RNAs including rRNAs, U6 snRNA and snoRNAs in the plasma microvesicles and their readily degradation in serum have been reported, which indicates that such non-coding RNAs may be inappropriate as endogenous controls (Chen et al., 2008). On a whole, more validations on the whole blood are warranted to identify circulating miRNA biomarkers that are of diagnostic value,

\section{REFERENCES}

Alix-Panabieres, C., and Pantel, K. (2013). Circulating tumor cells: liquid biopsy of cancer. Clin. Chem. 59, 110-118. doi: 10.1373/clinchem.2012.1 94258

Allard, W. J., Matera, J., Miller, M. C., Repollet, M., Connelly, M. C., Rao, C., et al. (2004). Tumor cells circulate in the peripheral blood of all major carcinomas but not in healthy subjects or patients with nonmalignant diseases. Clin. Cancer Res. 10, 6897-6904. doi: 10.1158/1078-0432.CCR-04-0378

Arroyo, J. D., Chevillet, J. R., Kroh, E. M., Ruf, I. K., Pritchard, C. C., Gibson, D. F., et al. (2011). Argonaute 2 complexes carry a population of circulating microRNAs independent of vesicles in human plasma. Proc. Natl. Acad. Sci. U.S.A. 108, 5003-5008. doi: 10.1073/pnas.1019055108

Bartel, D. P., and Chen, C.-Z. (2004). Micromanagers of gene expression: the potentially widespread influence of metazoan microRNAs. Nat. Rev. Genet. 5, 396-400. doi: $10.1038 / \operatorname{nrg} 1328$

Benz, F., Roderburg, C., Vargas Cardenas, D., Vucur, M., Gautheron, J., Koch, A., et al. (2013). U6 is unsuitable for normalization of serum miRNA levels in patients with sepsis or liver fibrosis. Exp. Mol. Med. 45, e42. doi: 10.1038/emm.2013.81

Bianchi, F., Nicassio, F., Marzi, M., Belloni, E., Dall'olio, V., Bernard, L., et al. (2011). A serum circulating miRNA diagnostic test to identify asymptomatic high-risk individuals with early stage lung cancer. EMBO Mol. Med. 3, 495-503. doi: 10.1002/emmm.201100154

Boeri, M., Verri, C., Conte, D., Roz, L., Modena, P., Facchinetti, F., et al. (2011). MicroRNA signatures in tissues and plasma predict development and prognosis of computed tomography detected lung cancer. Proc. Natl. Acad. Sci. U.S.A. 108, 3713-3718. doi: 10.1073/pnas.1100048108

Boyerinas, B., Park, S. M., Hau, A., Murmann, A. E., Peter, M. E. (2010). The role of let-7 in cell differentiation and cancer. Endocr. Relat. Cancer 17, F19-F36. doi: $10.1677 /$ erc-09-0184 coupled with a uniform and reliable internal control for data analysis.

CTCs are another type of "liquid biopsy" ideal for the diagnosis of NSCLC. Several issues remain to be addressed with respect to CTC enrichment and detection. Firstly, the platform of CTC detection technology combining marker-dependent (Cell Search) and marker-independent approaches (Isolation by size of epithelial tumor cells) has proven to be more sensitive than either of the two methods alone could be (Krebs et al., 2012). In addition, CTC-associated miRNAs, such as miR-21, can be applied to detect CTCs with an EMT phenotype (Ortega et al., 2015). We may hypothesize here CTC-associated miRNAs are likely to contain a larger amount of information underlying NSCLC cases, not merely being used as a marker for detection of CTCs. On the whole, future validation about such work is warranted before routine clinical applications are available.

\section{AUTHOR CONTRIBUTIONS}

LC, WC, and SW: Contributed the research concept and design; Moreover, LC: Finished critical revision of this article; JH: Finished the collection and assembly of data, and then she wrote the article; FM: Finished the drawing of the figure in this article.

\section{ACKNOWLEDGMENTS}

I would like to acknowledge that this project is supported by the Health and Medical Research Fund (HMRF, Project No. 02131026).

Chang, J., Nicolas, E., Marks, D., Sander, C., Lerro, A., Buendia, M. A., et al. (2004). miR-122, a mammalian liver-specific microRNA, is processed from hcr mRNA and may downregulate the high affinity cationic amino acid transporter CAT-1. RNA Biol. 1, 106-113. doi: 10.4161/rna.1.2.1066

Chen, X., Ba, Y., Ma, L., Cai, X., Yin, Y., Wang, K., et al. (2008). Characterization of microRNAs in serum: a novel class of biomarkers for diagnosis of cancer and other diseases. Cell Res. 18, 997-1006. doi: 10.1038/cr.2008.282

Chen, X., Wang, X., He, H., Liu, Z., Hu, J. F., and Li, W. (2015). Combination of circulating tumor cells with serum carcinoembryonic antigen enhances clinical prediction of non-small cell lung cancer. PLOS ONE 10:e0126276. doi: 10.1371/journal.pone.0126276

Choudhury, N. R., and Michlewski, G. (2012). Terminal loop-mediated control of microRNA biogenesis. Biochem. Soc. Trans. 40, 789-793. doi: 10.1042/BST20120053

Collino, F., Deregibus, M. C., Bruno, S., Sterpone, L., Aghemo, G., Viltono, L., et al. (2010). Microvesicles derived from adult human bone marrow and tissue specific mesenchymal stem cells shuttle selected pattern of miRNAs. PLoS ONE 5:e11803. doi: 10.1371/journal.pone.0011803

Dou, H., Wang, Y., Su, G., and Zhao, S. (2015). Decreased plasma let-7c and miR152 as noninvasive biomarker for non-small-cell lung cancer. Int. J. Clin. Exp. Med. 8, 9291-9298.

Fan, L., Qi, H., Teng, J., Su, B., Chen, H., Wang, C., et al. (2015). Identification of serum miRNAs by nano-quantum dots microarray as diagnostic biomarkers for early detection of non-small cell lung cancer. Tumour Biol. 37, 7777-7784. doi: 10.1007/s13277-015-4608-3

Gao, W., Liu, L., Lu, X., and Shu, Y. (2011). Circulating microRNAs: possible prediction biomarkers for personalized therapy of non-small-cell lung carcinoma. Clin. Lung Cancer 12, 14-17. doi: 10.3816/CLC.2011.n.001

Gherzi, R., Chen, C. Y., Trabucchi, M., Ramos, A., and Briata, P. (2010). The role of KSRP in mRNA decay and microRNA precursor maturation. Wiley Interdiscip. Rev. RNA 1, 230-239. doi: 10.1002/wrna.2 
Giallombardo, M., Chacártegui Borrás, J., Castiglia, M., Van Der Steen, N., Mertens, I., Pauwels, P., et al. (2016). Exosomal miRNA analysis in Non-small Cell Lung Cancer (NSCLC) patients' plasma through qpcr: a feasible liquid biopsy tool. J. Vis. Exp. doi: 10.3791/53900

Halvorsen, A. R., Bjaanaes, M., LeBlanc, M., Holm, A. M., Bolstad, N., Rubio, L., et al. (2016). A unique set of 6 circulating microRNAs for early detection of non-small cell lung cancer. Oncotarget 7, 37250-37259. doi: 10.18632/oncotarget.9363

Hanssen, A., Wagner, J., Gorges, T. M., Taenzer, A., Uzunoglu, F. G., Driemel, C., et al. (2016). Characterization of different CTC subpopulations in non-small cell lung cancer. Sci. Rep. 6:28010. doi: 10.1038/srep28010

Harb, W., Fan, A., Tran, T., Danila, D. C., Keys, D., Schwartz, M., et al. (2013). Mutational analysis of circulating tumor cells using a novel microfluidic collection device and qPCR assay. Trans. Oncol. 6, 528-538. doi: 10.1593/tlo. 13367

Hardingham, J. E., Kotasek, D., Farmer, B., Butler, R. N., Mi, J.-X., Sage, R. E., et al. (1993). Immunobead-PCR: a technique for the detection of circulating tumor cells using immunomagnetic beads and the polymerase chain reaction. Cancer Res. 53, 3455-3458.

Haus, D. L., Nguyen, H. X., Gold, E. M., Kamei, N., Perez, H., Moore, H. D., et al. (2014). CD133-enriched Xeno-free human embryonic-derived neural stem cells expand rapidly in culture and do not form teratomas in immunodeficient mice. Stem Cell Res. 13, 214-226. doi: 10.1016/j.scr.2014.06.008

Hou, J.-M., Greystoke, A., Lancashire, L., Cummings, J., Ward, T., Board, R., et al. (2009). Evaluation of circulating tumor cells and serological cell death biomarkers in small cell lung cancer patients undergoing chemotherapy. Am. J. Pathol. 175, 808-816. doi: 10.2353/ajpath.2009.090078

Huang, Y. (2012). A mirror of two faces: Lin28 as a master regulator of both miRNA and mRNA. Wiley Interdiscip. Rev. RNA 3, 483-494. doi: 10.1002/wrna.1112

Jemal, A., Center, M. M., DeSantis, C., and Ward, E. M. (2010). Global patterns of cancer incidence and mortality rates and trends. Cancer Epidemiol. Biomarkers Prev. 19, 1893-1907.doi: 10.1158/1055-9965.EPI-10-0437

Jemal, A., Clegg, L. X., Ward, E., Ries, L. A., Wu, X., Jamison, P. M., et al. (2004). Annual report to the nation on the status of cancer, 1975-2001, with a special feature regarding survival. Cancer 101, 3-27. doi: 10.1002/cncr.20288

Jorge, N., Marco, W., Madelyn, S. L., Dena, M., Lyudmila, B., Anand, K., et al. (2012). High-definition imaging of circulating tumor cells and associated cellular events in non-small cell lung cancer patients: a longitudinal analysis. Phys. Biol. 9:016004. doi: 10.1088/1478-3975/9/1/016004

Kong, D., Heath, E., Chen, W., Cher, M. L., Powell, I., Heilbrun, L., Li, Y., Ali, S., Sethi, S., Hassan, O. (2012). Loss of let-7 up-regulates EZH2 in prostate cancer consistent with the acquisition of cancer stem cell signatures that are attenuated by BR-DIM. PLoS ONE 7:e33729. doi: 10.1371/journal.pone.0033729

Krebs, M. G., Hou, J. M., Sloane, R., Lancashire, L., Priest, L., Nonaka, D., et al. (2012). Analysis of circulating tumor cells in patients with non-small cell lung cancer using epithelial marker-dependent and -independent approaches. J. Thorac. Oncol. 7, 306-315. doi: 10.1097/JTO.0b013e31823c5c16

Krol, J., Loedige, I., and Filipowicz, W. (2010). The widespread regulation of microRNA biogenesis, function and decay. Nat. Rev. Genet. 11, 597-610. doi: $10.1038 / \mathrm{nrg} 2843$

Lee, R. C., Feinbaum, R. L., and Ambros, V. (1993). The C. elegans heterochronic gene lin-4 encodes small RNAs with antisense complementarity to lin-14. Cell 75, 843-854. doi: 10.1016/0092-8674(93)90529-Y

Li, L., Chen, Y. Y., Li, S. Q., Huang, C., and Qin, Y. Z. (2015). Expression of miR148/152 family as potential biomarkers in non-small-cell lung cancer. Med. Sci. Monit. 21, 1155-1161. doi: 10.12659/MSM.892940

Li, Y., Liang, L., and Zhang, C. Y. (2013). Isothermally sensitive detection of serum circulating miRNAs for lung cancer diagnosis. Anal. Chem. 85, 11174-11179. doi: $10.1021 /$ ac403462f

Liljefors, M., Nilsson, B., Fagerberg, J., Ragnhammar, P., Mellstedt, H., and Frödin, J.-E. (2005). Clinical effects of a chimeric anti-EpCAM monoclonal antibody in combination with granulocyte-macrophage colony-stimulating factor in patients with metastatic colorectal carcinoma. Int. J. Oncol. 26, 1581-1589. doi: 10.3892/ijo.26.6.1581

Lund, E., Güttinger, S., Calado, A., Dahlberg, J. E., and Kutay, U. (2004). Nuclear export of microRNA precursors. Science, 303, 95-98. doi: 10.1126/science. 1090599
Ma, J., Lin, Y., Zhan, M., Mann, D. L., Stass, S. A., and Jiang, F. (2015). Differential miRNA expressions in peripheral blood mononuclear cells for diagnosis of lung cancer. Lab. Invest. 95, 1197-1206. doi: 10.1038/labinvest.2015.88

Ma, J., Liu, S., and Wang, Y. (2014). MicroRNA-21 and multiple myeloma: small molecule and big function. Med. Oncol. 31, 94. doi: 10.1007/s12032-014-0094-5

Man, Y., Cao, J., Jin, S., Xu, G., Pan, B., Shang, L., et al. (2014). Newly identified biomarkers for detecting circulating tumor cells in lung adenocarcinoma. Tohoku J. Exp. Med. 234, 29-40. doi: 10.1620/tjem.234.29

Markou, A., Sourvinou, I., Vorkas, P. A., Yousef, G. M., and Lianidou, E. (2013). Clinical evaluation of microRNA expression profiling in non-small cell lung cancer. Lung Cancer 81, 388-396. doi: 10.1016/j.lungcan.2013.05.007

Mascalchi, M., Falchini, M., Maddau, C., Salvianti, F., Nistri, M., Bertelli, E., et al. (2016). Prevalence and number of circulating tumour cells and microemboli at diagnosis of advanced NSCLC. J. Cancer Res. Clin. Oncol. 142, 195-200. doi: $10.1007 / \mathrm{s} 00432-015-2021-3$

Mayr, F., and Heinemann, U. (2013). Mechanisms of Lin28-mediated miRNA and mRNA regulation-a structural and functional perspective. Int. J. Mol. Sci. 14, 16532-16553. doi: 10.3390/ijms140816532

Michlewski, G., and Cáceres, J. F. (2010). Antagonistic role of hnRNP A1 and KSRP in the regulation of let-7a biogenesis. Nat. Struct. Mol. Biol. 17, 1011-1018. doi: 10.1038/nsmb. 1874

Michlewski, G., Guil, S., Semple, C. A., and Cáceres, J. F. (2008), Posttranscriptional regulation of miRNAs harboring conserved terminal loops. Mol. Cell 32, 383-393. doi: 10.1016/j.molcel.2008.10.013

Mitchell, P. S., Parkin, R. K., Kroh, E. M., Fritz, B. R., Wyman, S. K., PogosovaAgadjanyan, E. L., et al. (2008). Circulating microRNAs as stable blood-based markers for cancer detection. Proc. Natl. Acad. Sci. U.S.A. 105, 10513-10518. doi: 10.1073/pnas.0804549105

Muinelo-Romay, L., Vieito, M., Abalo, A., Nocelo, M. A., Barón, F., Anido, U., et al. (2014). Evaluation of circulating tumor cells and related events as prognostic factors and surrogate biomarkers in advanced NSCLC patients receiving first-line systemic treatment. Cancers (Basel). 6, 153-165. doi: $10.3390 /$ cancers 6010153

O’Flaherty, J. D., Gray, S., Richard, D., Fennell, D., O’Leary, J. J., Blackhall, F. H., et al. (2012). Circulating tumour cells, their role in metastasis and their clinical utility in lung cancer. Lung Cancer 76, 19-25. doi: 10.1016/j.lungcan.2011.10.018

Okamura, K., Chung, W. J., and Lai, E. C. (2008). The long and short of inverted repeat genes in animals: microRNAs, mirtrons and hairpin RNAs. Cell Cycle 7, 2840-2845. doi: 10.4161/cc.7.18.6734

Ortega, F. G., Lorente, J. A., Garcia Puche, J. L., Ruiz, M. P., Sanchez-Martin, R. M., de Miguel-Perez, D., et al. (2015). miRNA in situ hybridization in circulating tumor cells-MishCTC. Sci. Rep. 5:9207. doi: 10.1038/srep09207

Ozretić, L., Heukamp, L. C., Odenthal, M., and Buettner, R. (2012). The role of molecular diagnostics in cancer diagnosis and treatment. Onkologie 35(Suppl. 1), 8-12. doi: 10.1159/000334823

Park, J.-K., Lee, E. J., Esau, C., and Schmittgen, T. D. (2009). Antisense inhibition of microRNA-21 or-221 arrests cell cycle, induces apoptosis, and sensitizes the effects of gemcitabine in pancreatic adenocarcinoma. Pancreas 38, e190-e199. 10.1097/MPA.0b013e3181ba82e1

Parkinson, D. R., Dracopoli, N., Petty, B. G., Compton, C., Cristofanilli, M., Deisseroth, A., et al. (2012). Considerations in the development of circulating tumor cell technology for clinical use. J. Transl. Med. 10, 1. doi: 10.1186/14795876-10-138

Petriella, D., Galetta, D., Rubini, V., Savino, E., Paradiso, A., Simone, G., et al. (2013). Molecular profiling of thin-prep FNA samples in assisting clinical management of non-small-cell lung cancer. Mol. Biotechnol. 54, 913-919. doi: 10.1007/s12033-012-9640-6

Powrózek, T., Krawczyk, P., Kowalski, D. M., Winiarczyk, K., OlszynaSerementa, M., and Milanowski, J. (2015). Plasma circulating microRNA-944 and microRNA-3662 as potential histologic type-specific early lung cancer biomarkers. Trans. Res. 166, 315-323. doi: 10.1016/j.trsl.2015.05.009

Rabinowits, G., Gerçel-Taylor, C., Day, J. M., Taylor, D. D., and Kloecker, G. H. (2009). Exosomal microRNA: a diagnostic marker for lung cancer. Clin. Lung Cancer 10, 42-46. doi: 10.3816/CLC.2009.n.006

Raponi, M., Dossey, L., Jatkoe, T., Wu, X., Chen, G., Fan, H., et al. (2009). MicroRNA classifiers for predicting prognosis of squamous cell lung cancer. Cancer Res. 69, 5776-5783. doi: 10.1158/0008-5472.CAN-09-0587 
Scagliotti, G. V., Fossati, R., Torri, V., Crinò, L., Giaccone, G., Silvano, G., et al. (2003). Randomized study of adjuvant chemotherapy for completely resected stage I, II, or IIIA non-small-cell lung cancer. J. Natl. Cancer Inst. 95, 1453-1461. doi: 10.1093/jnci/djg059

Shen, J., Todd, N. W., Zhang, H., Yu, L., Lingxiao, X., Mei, Y., et al. (2011). Plasma microRNAs as potential biomarkers for non-small-cell lung cancer. Lab. Invest. 91, 579-587. doi: 10.1038/labinvest.2010.194

Si, M. L., Zhu, S., Wu, H., Lu, Z., Wu, F., and Mo, Y. Y. (2007). miR-21-mediated tumor growth. Oncogene 26, 2799-2803. doi: 10.1038/sj.onc.1210083

Silva, J., Garcia, V., Zaballos, Á., Provencio, M., Lombardía, L., Almonacid, L., et al. (2011). Vesicle-related microRNAs in plasma of nonsmall cell lung cancer patients and correlation with survival. Eur. Respir. J. 37, 617-623. doi: 10.1183/09031936.00029610

Trang, P., Wiggins, J. F., Daige, C. L., Cho, C., Omotola, M., Brown, D., et al. (2011). Systemic delivery of tumor suppressor microRNA mimics using a neutral lipid emulsion inhibits lung tumors in mice. Mol. Ther. 19, 1116-1122. doi: $10.1038 / \mathrm{mt} .2011 .48$

Travis, W. D., Brambilla, E., Noguchi, M., Nicholson, A. G., Geisinger, K. R., Yatabe, Y., et al. (2011). International association for the study of lung cancer/American Thoracic Society/European Respiratory Society international multidisciplinary classification of lung Adenocarcinoma. J. Thorac. Oncol. 6, 244-285. doi: 10.1097/JTO.0b013e318206a221

Valadi, H., Ekström, K., Bossios, A., Sjöstrand, M., Lee, J. J., and Lötvall, J. O. (2007). Exosome-mediated transfer of mRNAs and microRNAs is a novel mechanism of genetic exchange between cells. Nat. Cell Biol. 9, 654-659. doi: 10.1038/ncb1596

Vickers, K. C., Palmisano, B. T., Shoucri, B. M., Shamburek, R. D., and Remaley, A. T. (2011). MicroRNAs are transported in plasma and delivered to recipient cells by high-density lipoproteins. Nat. Cell Biol. 13, 423-433. doi: 10.1038/nc b2210

Volinia, S., Calin, G. A., Liu, C. G., Ambs, S., Cimmino, A., Petrocca, F., et al. (2006). A microRNA expression signature of human solid tumors defines cancer gene targets. Proc. Natl. Acad. Sci. U.S.A. 103, 2257-2261. doi: 10.1073/pnas.0510565103

Wang, K., Zhang, S., Weber, J., Baxter, D., and Galas, D. J. (2010). Export of microRNAs and microRNA-protective protein by mammalian cells. Nucleic Acids Res. 38, 7248-7259. doi: 10.1093/nar/gkq601

Wang, R. J., Zheng, Y. H., Wang, P., and Zhang, J. Z. (2015). Serum miR-125a-5p, miR-145 and miR-146a as diagnostic biomarkers in non-small cell lung cancer. Int. J. Clin. Exp. Pathol. 8, 765-771.
Westholm, J. O., and Lai, E. C. (2011). Mirtrons: microRNA biogenesis via splicing. Biochimie 93, 1897-1904. doi: 10.1016/j.biochi.2011.06.017

Wood, D. E., Eapen, G. A., Ettinger, D. S., Hou, L., Jackman, D., Kazerooni, E., et al. (2012). Lung cancer screening. J. Natl. Compr. Cancer Netw. 10, 240-265.

Wozniak, M. B., Scelo, G., Muller, D. C., Mukeria, A., Zaridze, D., and Brennan, P. (2015). Circulating microRNAs as non-invasive biomarkers for early detection of non-small-cell lung cancer. PLoS ONE 10:e0125026. doi: 10.1371/journal.pone.0125026

Wu, S., Liu, S., Liu, Z., Huang, J., Pu, X., Li, J., et al. (2015). Classification of circulating tumor cells by epithelial-mesenchymal transition markers. PLoS ONE 10:e0123976. doi: 10.1371/journal.pone.0123976

Xiang, M., Zeng, Y., Yang, R., Xu, H., Chen, Z., Zhong, J., et al. (2014). U6 is not a suitable endogenous control for the quantification of circulating microRNAs. Biochem. Biophys. Res. Commun. 454, 210-214. doi: 10.1016/j.bbrc.2014.10.064

Yang, M., Shen, H., Qiu, C., Ni, Y., Wang, L., Dong, W., et al. (2013). High expression of miR-21 and miR-155 predicts recurrence and unfavourable survival in non-small cell lung cancer. Eur. J. Cancer 49, 604-615. doi: 10.1016/j.ejca.2012.09.031

Young, R., Pailler, E., Billiot, F., Drusch, F., Barthelemy, A., Oulhen, M., et al. (2012). Circulating tumor cells in lung cancer. Acta Cytol. 56, 655-660. doi: $10.1159 / 000345182$

Zandberga, E., Kozirovskis, V., Abols, A., Andrejeva, D., Purkalne, G., and Line, A. (2013). Cell-free microRNAs as diagnostic, prognostic, and predictive biomarkers for lung cancer. Genes Chromosomes Cancer 52, 356-369. doi: 10.1002 /gcc. 22032

Zernecke, A., Bidzhekov, K., Noels, H., Shagdarsuren, E., Gan, L., Denecke, B., et al. (2009). Delivery of microRNA-126 by apoptotic bodies induces CXCL12-dependent vascular protection. Sci. Signal. 2, ra81-ra81. doi: $10.1126 /$ scisignal.2000610

Conflict of Interest Statement: The authors declare that the research was conducted in the absence of any commercial or financial relationships that could be construed as a potential conflict of interest.

Copyright (c) 2016 Hou, Meng, Chan, Cho and Wong. This is an open-access article distributed under the terms of the Creative Commons Attribution License (CC BY). The use, distribution or reproduction in other forums is permitted, provided the original author(s) or licensor are credited and that the original publication in this journal is cited, in accordance with accepted academic practice. No use, distribution or reproduction is permitted which does not comply with these terms. 